\title{
Analysis of the Ecological Management and Control Strategy of the Third Party Sellers on the Electronic Business Platform of JD.com
}

\author{
Xiaowei Zhu ${ }^{1,2}$ and Li Yuan ${ }^{2}$ \\ ${ }^{1}$ Wuhan Technology and Business University, China, Hubei, Wuhan, 430065 \\ ${ }^{2}$ Hubei Business Service Development Research Center, China, Hubei, Wuhan, 430065
}

Keywords: Electronic commerce; Electronic business logistics; Management and control strategy

\begin{abstract}
At present, the rapid growth of e-commerce transactions in China, the innovation of its operation model has become increasingly active, e-commerce presenting a multi-level, diversified development trend. At the same time electronic business platform for the rapid growth of business, the problem of management platform has come out. Many businesses use platform vulnerabilities and sell fake, false trading, prevarication, which is contrary to honest business and professional ethics of irregularities. In this paper, taking JD.com business platform as an example, combine with their own practice experience in the company's quality control department, through analyzing the problems existing in the existing management and control strategy of the JD.com platform, put forward the corresponding rectification suggestions, promote the platform to build a good seller's ecological environment.
\end{abstract}

\section{Introduction}

According to the China e-commerce research center statistics, from the scale of the transaction to view, Since 2009 since our country electronic commerce as a whole has maintained rapid development momentum, annual growth with $2 \sim 3$ times the rate of gdp $7 \% \sim 9 \%$. By the end of 2012, China's e-commerce market transactions amounted to seven trillion and eight hundred and fifty billion yuan with an increase of 30.8\%; By the end of 2013, China's e-commerce transaction volume has broken the barrier of 10 trillion yuan, up to more than ten trillion and two hundred billion yuan, an increase of $29.9 \%$, e-commerce entering the stage of scale development. From the contribution of the economy to view, since 2009, China's e-commerce contribution to the economy has become increasingly prominent, the proportion of its transactions accounted for GDP showed a trend of growth over the years. From 10.9\% growth in 2009 to in 2013 of $29.9 \%$, an increase of nearly 1.7 times has become a leading force in the development of China's economy. But frankly, with the rapid development of the electricity industry and at the same time the rapid growth in the number of sellers on business platform, appearing cohabitation situation, many businesses use platform vulnerabilities and sell fake, false trading, prevarication, contrary to honest business and professional ethics of irregularities, which have a serious impact on the overall development of electricity industry.

\section{Ecological Management Overview of Third Party Seller}

The Definition of Ecological Management and Control of Third Party Sellers. The seller ecological is refers to the state of under certain market environment, business survival and development. Seller's ecological management and control refers to the interaction among economics, ecology and sociology, which is a kind of management for the system in a way that can protect the long-term ecological sustainability and diversity of the market environment. Taking JD.com as an example, seller's ecological management and control in a broad sense refers to all aspects of the platform for the management and control from the seller's enter to withdraw . Merchants from entering to exit platform every link has its corresponding norms and standards, the platform to achieve the purpose of managing the business for the platform to achieve the purpose of managing 
the business; From the narrow sense, it is refers that the platform through the deduction of fines and other forms to limit sell fake, false trading, broken promises, such as a series of violations.

The Significance of Ecological Management and Control of Third Party Sellers. Development of a reasonable market ecosystem and benign market competition is able to produce ecological synergies through good cooperation and communication, to create a higher than the cost of capital gains, and bring lasting ecological value and influence.

\section{The Problems on Jingdong Platform for the Third Party Seller Ecological Control}

Ecological Management Control Problems of Third Party Seller. Before entering; in the aspect of merchants settled, JD. Comhas a professional audit team with qualification the, for each settled merchants are in accordance with the JD.com investment standards and various industry standards and other relevant requirements, strict audit, to ensure that businesses are fully qualified. In terms of the contract, the JD.com signed a series of product quality assurance contracts with businesses, to take the most stringent measures to deal with fake violations. Although JD.com on the sale of counterfeit goods is zero tolerance", on the platform, also can't avoid the sale of fake, Even with a phenomenon that a merchant on the platform at the same time open dozens of different types of shops. The appearance of this phenomenon shows that JD.com has a professional qualification audit team, but still have a lot of defects need to make up. In addition, JD.com platform brands have a lot but many did not settled down to the JD.com, such as NIKE, a large brand settling on a platform is the greatest recognition to their quality and service, and that how to attract more big brands settled in the JD.com is a platform to consider the problem.

During Settling; Illegal management is the most important part of the business management, but also the most important part of the seller's ecological control. From August 18, 2015 it have been 8 months in the JD.com quality control department, in the period, I has a general understanding the operation process of seller in the JD.com platform. The business violations can be seen almost every day, starting from the internship experience in the second half of the year, which can be summed up the JD. Complatform businesses violations are mainly the following categories:

Sale of counterfeit goods; the sale of counterfeit goods: refers to behavior that the seller sell counterfeit and pirated goods

False trading; False trading: refers to the seller through improper means to increase sales in the JD.com open platform sales of goods or increase shop mark (that is, "speculation" behavior), which acts of prejudice to the rights and interests of buyers. This "speculation" behavior mainly includes but not limits to the following forms: self-buy and self-rating, malicious change prices, replacement of goods, third party speculation, empty boxes, etc.

Improper use of others' rights; improper use of other people's rights: refers to the infringement of the trademark in addition to counterfeiting, in addition to piracy, copyright infringement, as well as the infringement of the legitimate rights and interests of other legal rights and interests of others.

In 2015, the number of violations that merchants improperly use other's right is about 3000 times in JD.com platform, accounting for 3\% of the total number of violations. What can be seen from the data is that JD.com platform because of the improper use of the others illegal rights businesses are not many, but improper use of rights of others is one of serious violations. JD.com platform for improper use of the rights of others particularly serious impact on the circumstances, according to a serious violation of the treatment of each buckle 100 points

After Settling; Businesses need to pay the quality assurance when the most settles in the platform, when merchants withdraw from the platform, the warranty will be returned to the business, however, this process has been a problem that many of the list is still not processed for a long time, and then the cumulative more. Merchant once choose no longer to cooperate with the platform in addition to the issue of quality assurance. In fact, there is no relationship with the platform, however, in this piece of the process will be very slow, which leads that business will not stop the mail call to refund. 
on the other hand, it is not good enough for the JD.com in the service, Whether it is assigned to the platform or exit platform, JD.com should holds the principle of the supremacy of service, no matter what step should not be relaxed, even if in the end should give themselves a good reputation, so the system upgrades and continuous optimization is very important.

\section{Ecological Management Recommendations on the JD.com's Electronic Business Platform for the Third Side of the Seller's}

JD.com since its inception, has been to authentic licensed the concept based on the electric business, making every effort to bring the most secure shopping environment for consumers, JD.com requirements on the quality of goods as to business requirements. So how can give businesses to provide the most secure shopping environment, which requires the platform must have a good seller's ecological environment, the seller does not violate the rules of fraud, of course, consumers buy the rest assured that the purchase of comfort. How to control the third party seller? This requires the full implementation of the business sector quality control, investment, quality audit, after sales, research and development and other departments to support cooperation, and jointly with the government regulators to create a good ecological environment of the seller. How should all departments be to cooperate with it?

Qualification Management. JD.com has a professional qualification audit team that in addition play their own ability should also focus on the study of the system. The perfection of the system is of great significance to improve the accuracy and efficiency, and some things cannot rely on the subjective judgment to judge the ability, must rely on the system. In addition to that the audit department should cooperate closely with the investment sector, this will be able to avoid the phenomenon of the same business open the multi category stores on platform, although revenue is important, it is important to reduce the rate of violation. Qualification audit must grasp the first step, do not let fake and shoddy businesses to enter the platform, so even if there are fake and shoddy businesses that it is a very small number.

Big Brand Settled. JD.com platform can cut down the threshold for the business of large brands, for example, to reduce the margin charges, charges standard. Big brand is settled down on the platform which certain the best quality and service, so we must increase efforts to attract large brands of merchants settled in the JD.com.

Illegal Management. Sale of counterfeit goods; JD.com for counterfeit goods holds a "zero tolerance" principle, the Jingdong platform for the sale of counterfeit goods and the circumstances are serious, each deduction of 100 points. To protect the rights and interests of consumers, for the seller in violation of the circumstances, JD.com to take market control measures as the seriousness of the circumstances (including but not limiting to stop shops, restrictions on the release of goods, public warning, closing shops, etc.). The most direct way to eliminate counterfeit goods is to strengthen the quality of the audit, At the beginning of a good first step, do not let fake and shoddy businesses to enter the platform, and then auxiliary manual investigation, once confirmed immediately take measures to take off the shelf, and in accordance with the actual situation of businesses to be punished.

False trading; JD.com for the false trading line also holds the principle of "zero tolerance", in addition to the strict management and rule of reward and punishment, JD.com should make use of advanced technology to form a complete reliable anti technology barrier through the data, also as the risk control "Skynet", to protect the user's best interest. At present, the recognition accuracy of the JD.com anti scalping system has reached more than $99 \%$. But it is not the best choice to punish businesses to regulate the behavior of businesses, which on the contrary may allow businesses to complain about the heart, do not want to continue to cooperate with the JD.com, therefore, in the continuous improvement of the system on the basis of skynet, for businesses to do a good place, we should also reward. Rewards and punishments are clear to allow businesses to do a good work to 
get a reward, rather than always complaining about why to punish them, the punishment is only a means, but the guide is more important, businesses get the right guidance, get the proper reward will not have the illegal.

False propaganda; For false propaganda, it can take the form of artificial investigation and system investigation to reduce the violation rate, each investigation of a commodity, using the results of the investigation to inform the business and urge businesses to improve, so that a round of the investigation down, the business of the illegal rate will decline naturally. However, the existing technology can only rely on human investigation, so there will be a problem: human consumption is more, the duration of the cycle is too long, the cost is too high, so the system is very important to research and development. The investigation needs to rely on a strong system, so that it can improve efficiency, but also to avoid the errors caused by subjective judgments.

Over issue information; the most common JD.com platform spam is the title and the main figure of illegal violations. JD.com on the platform above the commodity has a unified title specification and various categories of different main chart specification. Businesses need to fill in the title accordance with their own category of specifications and upload the main map of the commodity, if not in compliance with the specification; it will be identified as not in line with the provisions of the information.

Platform Rule Management. Quality control department in addition to cut points, open a ticket and fine for the business, more consideration should be given to how to reduce these irregularities, rather than to force the business to comply with these laws and regulations, if the business can be their own to comply with these rules that a good ecological environment came into being. How can allow businesses to take the initiative to comply with these rules?

Set shortcut button on the regular page; first you have to let him know the existence of these rules, and now enter the rules of the page needing to click Merchants settled on the bottom of the Jingdong, which is a very bad user experience. If I was a businessman, I might not look at this page, so you need to set a rule on the front page of the JD.com to facilitate business Click to read.

Training for Merchant settling; Secondly, businesses know the existence of this page, but he do not take the initiative to see, which requires investment sector in the beginning merchants settling to have an online training and settled down only by passing examination. A settling note manual Can also publish for businesses to learn in the future. The form of examination for businesses can allow businesses to be quickly familiar with the rules of understanding platform, pay attention to avoid violations, thereby reducing the rate of illegal businesses.

Establish incentive mechanism; After merchants settled in, on the platform the reward and punishment mechanism seems to have been punished businesses and don't see the reward mechanism, which is a big problem, businesses should be punished violations ,but for businesses to do a good place, we should also reward. Rewards and punishments are clear to allow businesses to do a good work to get a reward, rather than always complaining about why to punish them, the punishment is only a means, but the guide is more important, businesses get the right guidance, get the proper reward will not have the illegal.

\section{Summary}

JD.com from the establishment of the electronic business platform to now is actually a very perfect platform, from the beginning of a single sale to the current diversified JD.com has been in progress. Constantly challenge more new things from the fields that they are good at, the open of Pop platform brought huge benefits but also brings a lot of problems to JD.com. Poor seller's ecological environment is urgent need to solve for platform, no matter how complex the problem can be traced back to the source, from the beginning to analyze the problem, can always find a way to solve the problem. JD.com electronic business platform for the framework of the seller's ecological management and control is very complete, needing to do more details problem, as the old words: 
the details of the success or failure of the decision. JD.com platform needs to grasp the overall situation of the premise, pay attention to the detail adjustment, so as to establish a good seller of the ecological environment, and promote the development of the platform and the business of common and harmonious development.

\section{Acknowledgements}

Fund Project: Hubei Provincial Collaborative Innovation Center for Modern logistics and business (Project No.2011A201307)

Fund Project: MOE (Ministry of Education in China) Project of Humanities and Social Sciences (Project No.14YJCZH154)

Funding team: Wuhan Technology and Business University Academic Team ( Project No. XSTD2015004)

\section{References}

[1] Li Boqun. Research on the present situation and Prospect of electronic commerce in China [J].Research world, 2015, 01:15-18.

[2] Ye Jiali.The present situation and problems of the development of electronic commerce in China [J].Business economy, 2010, 07:35-37.

[3] Liu Ying, Lou Cequn.Application status and utilization strategy of third party electronic commerce platform [J].Library and information work, 2010,14:131-135.

[4] Ren Jin Fang. Electronic commerce ecosystem and its development environment [J]. Journal of Minjiang University), 2010, 04:62-65.

[5] Wang Muxin. New trend of global e-commerce development and the innovation and development of China's e-commerce [D].Jilin University, 2015

[6] Liu Ying. Research on optimization mechanism and measures of e-commerce ecological environment [D]. Central China Normal University, 2011

[7] Guan Rongwei. Analysis on the choice of the third party e-commerce platform for small and medium sized enterprises [J]. Market Forum, 2011, 07: 34-35.

[8] Liu Jie. Research on the third party e-commerce model of small and medium sized enterprises [J].Journal of Liaoning Vocational College of Economics (Liaoning Institute of economic management and management), 2007,01:58-59.

[9] Zhang Tao. Research on the competitive advantage of small and medium sized enterprises cluster based on the third party e-commerce platform [D]. Yunnan University of Finance and Economics, 2012

[10] Jiao Xinlong. Study on the new development model of modern logistics industry based on electronic commerce [D]. Xiangtan University, 2005

[11]Liu Zongmei, Li Zhiyu. Construction of legal environment of electronic commerce [J]. Journal of Liaoning Administration College, 2008, 02:30-31. 